\title{
SERINC2-knockdown inhibits proliferation, migration and invasion in lung adenocarcinoma
}

\author{
YUAN ZENG ${ }^{1,2^{*}}$, DAKAI XIAO ${ }^{1,2^{*}}$, HUIMING HE $^{1,2}$, JIAXI HE $^{1,2}$, HUI PAN $^{1,2}$, \\ WEI YANG ${ }^{3}$, YAOQI CHEN ${ }^{1,2}$ and JIANXING $\mathrm{HE}^{1,2}$
}

\begin{abstract}
${ }^{1}$ Department of Thoracic Surgery, The First Affiliated Hospital of Guangzhou Medical University, Guangzhou, Guangdong 510120; ${ }^{2}$ State Key Laboratory of Respiratory Diseases and National Clinical Research Center for Respiratory Disease and Guangzhou Institute of Respiratory Diseases, Guangzhou, Guangdong 510000; ${ }^{3}$ Department of Cardiothoracic Surgery, The Second Affiliated Hospital of Nanchang University, Nanchang, Jiangxi 330008, P.R. China
\end{abstract}

Received May 2, 2018; Accepted July 16, 2018

DOI: $10.3892 / \mathrm{ol} .2018 .9403$

\begin{abstract}
Serine incorporator 2 (SERINC2) is a member of the SERINC family of transmembrane proteins that incorporate serine into membrane lipids during synthesis. In the present study, the biological function of SERINC2 in lung adenocarcinoma cells was investigated. The data from a previous study and the publicly available Oncomine database were analysed regarding the expression levels of SERINC2 mRNA in lung adenocarcinoma. A lentiviral-based short hairpin RNA (shRNA) was used to suppress SERINC2 expression in lung cancer cells. The effect of SERINC2 expression on lung cancer proliferation was determined using cell counting kit- 8 and colony formation assays. The influence on invasion and migration was examined in vitro using Transwell and wound-healing assays, respectively. Phosphorylated protein kinase B (p-AKT) expression levels were assessed by immunoblotting. According to a previous study and Oncomine, expression levels of SERINC2 mRNA are significantly upregulated in tumour tissues compared with those in healthy tissues in patients with lung adenocarcinoma. SERINC2-knockdown by lentiviral-based shRNA inhibited the proliferation, migration and invasion of the H1650 and A549 cells. In addition, p-AKT expression levels were significantly decreased following SERINC2-knockdown. In conclusion, SERINC2-knockdown suppresses lung adenocarcinoma proliferation, migration and invasion through a mechanism that may be associated with phosphatidylinositol 3-kinase/AKT signalling. Based on these
\end{abstract}

Correspondence to: Dr Jianxing He, Department of Thoracic Surgery, The First Affiliated Hospital of Guangzhou Medical University, 151 Yanjiang Road, Guangzhou, Guangdong 510120, P.R. China

E-mail: drjianxing.he@gmail.com

*Contributed equally

Key words: serine incorporator 2, lung cancer, protein kinase B findings, SERINC2 serves an important role in the progression of lung adenocarcinoma.

\section{Introduction}

Lung cancer has been reported as the leading cause of cancer-associated mortality worldwide, with $\sim 1.8$ million new cases and 1.5 million mortalities attributed to this disease in 2012 worldwide (1). Non-small cell lung cancer (NSCLC) has been reported to account for $\sim 85 \%$ of all cases of lung cancer (2) with a 5-year overall survival rate of $\sim 15 \%$ (3). Lung adenocarcinoma has been reported as the most commonly diagnosed histological type of NSCLC, with a mortality rate of $>500,000$ individuals per year (4).

Therapies targeting a wide variety of activated oncogenes, translocations or fusions, including epidermal growth factor receptor (5), ALK receptor tyrosine kinase (6), KRAS proto-oncogene GTPase (7) and ROS proto-oncogene 1 receptor tyrosine kinase (8), have been demonstrated to serve a crucial role in the progress of treating patients with lung cancer. Nonetheless, further development of novel and effective therapeutic targets for treating lung cancer is required. In our previous study, gene profiles between lung adenocarcinoma tissues and adjacent healthy lung tissues were compared using whole-genome sequencing and a number of differentially expressed genes (DEGs) were identified (9). Among the DEGs, serine incorporator 2 (SERINC2), a member of the SERINC family of transmembrane proteins that incorporate serine into membrane lipids during synthesis, was of particular note (10).

Although the family is highly conserved among eukaryotes, none of the SERINC family members (SERINC1-5) display amino acid homology to other proteins. According to Inuzuka et al (10), SERINC proteins have an essential function in regulating the biosynthesis of multiple membrane lipids, including phosphatidylserine and sphingolipid molecules. Furthermore, phosphatidylserine (11-13) and sphingolipids (14-16) have been indicated to serve critical roles in cancer development and progression. Ren et al (17) demonstrated that small interfering RNA (siRNA)-mediated knockdown of SERINC1 in hepatocellular carcinoma inhibits cell cycle progression by transcriptional activation of $\mathrm{p} 21$. 
In 2003, Player et al (18) initially identified human SERINC2, which was referred to as TDE2. The deduced 456-amino acid protein has 11 putative transmembrane helices, a cysteine-rich region near the $\mathrm{N}$-terminus, followed by a phenylalanine-rich region and a central conserved myelocytomatosis oncogene-type helix-loop dimerization domain. It has been demonstrated by reverse transcription-quantitative polymerase chain reaction (RT-qPCR) that SERINC2 transcript levels are increased in NSCLC compared with the levels in non-malignant tissues (18). In addition, in situ hybridization assays have indicated that lung tumours express increased expression levels of SERINC2 transcripts compared with adjacent non-malignant regions (18). However, the role of SERINC2 in cancer remains unknown. In the present study, the effects of SERINC2-knockdown on proliferation, migration and invasion of lung adenocarcinoma cell lines was investigated.

\section{Materials and methods}

Analysis of Oncomine data. The Oncomine database, an online cancer microarray database that enables multiple comparisons of gene expression levels in DNA and RNA reported in different studies, was used for analysing the expression pattern of SERINC2 in lung adenocarcinoma (https://www.oncomine. org). The SERINC2 gene was queried and the results were filtered by selecting 'lung adenocarcinoma' and 'Cancer vs. Normal Analysis'. Normal lung tissue was used as the control group. Comparative statistical analysis was conducted using Oncomine algorithms. $\mathrm{P}<0.01$ was considered to indicate a statistically significant difference. Details of standardized normalization techniques and statistical calculations are provided on the Oncomine platform.

Cell line culture and transfection. Human lung cancer H1650 and A549 cell lines were purchased from the Shanghai Institutes for Biological Sciences (Shanghai, China) and cultured in Dulbecco's modified Eagle's medium, supplemented with $10 \%$ foetal bovine serum (Gibco; Thermo Fisher Scientific, Inc., Waltham, MA, USA), $100 \mathrm{U} / \mathrm{ml}$ penicillin and $100 \mathrm{mg} / \mathrm{ml}$ streptomycin. All cells were maintained at $37^{\circ} \mathrm{C}$ with $5 \% \mathrm{CO}_{2}$.

Two short hairpin RNAs (shRNAs) targeting SERINC2 were designed and constructed by Sangon Biotech Co., Ltd. (Shanghai, China). The following sequences were inserted into the Lentiviral shRNA Vector (Forevergen Biotechnology, Guangzhou, China): sh1, 5'-TGCGCCTCATCTTCACGT TCTTCTCAAGAGGAAGAACGTGAAGATGAGGCGTTT TTC-3' and sh2, 5'-TGTGGTCAGCCCTATCCAGTATCT CAAGAGGATACTGGATAGGGCTGACCATTTTTC-3'. Lipofectamine $2000^{\circledR}$ (Invitrogen; Thermo Fisher Scientific, Inc.) was used for transfection, according to the manufacturer's protocols. Stable cell lines expressing SERINC2-shRNA were generated by infection with lentiviruses $(1 \mu \mathrm{l}$; Forevergen Biotechnology) produced in 293 cells and selection of stable clones with $0.5 \mu \mathrm{g} / \mathrm{ml}$ puromycin for 10 days, then subsequent experiments were performed.

$R T-q P C R$. Total RNA was extracted from H1650 and A549 cells using TRIzol reagent (Invitrogen; Thermo Fisher Scientific, Inc.). The concentration and quality of total RNA samples were determined by spectrophotometry and agarose gel electrophoresis, respectively. cDNA templates were synthesized from $1 \mu \mathrm{g}$ total RNA using a Reverse Transcription system (Promega Corporation, Madison, WI, USA), according to the manufacturer's protocols. qPCR was subsequently performed using GoTaq qPCR Master mix (Promega Corporation ), according to the manufacturer's protocols. The following thermocycling conditions were used for the qPCR: $95^{\circ} \mathrm{C}$ for $3 \mathrm{~min}$, followed by 40 cycles of $95^{\circ} \mathrm{C}$ for $3 \mathrm{sec}$ and $60^{\circ} \mathrm{C}$ for $30 \mathrm{sec}$. The quality of the PCR products was assessed using a post-PCR melting curve and $\beta$-actin served as the internal control. Relative mRNA expression levels were quantified using the $2^{-\Delta \Delta C q}$ method (19). The following primers were used: SERINC2 sense, 5'-TGGTGCTGCTCATCGACTTT-3' and antisense, 5'-TGAAGAAGAAGAGGCCTGCG-3'; and $\beta$-actin sense, 5'-AGAAGAGCTACGAGCTGCCTGACG-3' and antisense, 5'-GGACTCCATGCCACGGAAGGAA-3'.

Immunoblotting. Following harvesting of cells, total cellular proteins were extracted with radioimmunoprecipitation assay lysis buffer containing protease inhibitors (Sangon Biotech Co., Ltd.). Protein concentrations were measured using the Bradford assay(Bio-RadLaboratories,Inc.,Hercules,CA,USA), according to the manufacturer's protocols with bovine serum albumin as the standard. Equal amounts of protein extracts (80-120 $\mu \mathrm{g}$, dependent on experiment) from all samples were separated by $10 \%$ SDS-PAGE and subsequently transferred to a polyvinylidene fluoride membrane. The membrane was blocked with 5\% skimmed milk dissolved in Tris-buffered saline containing $0.1 \%$ Tween-20 for $1 \mathrm{~h}$ at room temperature followed by incubation using anti-SERINC2 (1:1,000 dilution; cat. no. ab134312; Abcam, Cambridge, MA, USA), anti-phospho-AKT (1:1,000 dilution; ser 473, cat. no. 4058; Cell Signaling Technology, Inc., Danvers, MA, USA), anti-AKT (1:1,000 dilution; cat. no. 9272; Cell Signaling Technology, Inc.) and $\beta$-actin $(1: 2,000$ dilution; cat. no. A001041; Sangon Biotech Co., Ltd.) primary antibodies overnight at $4^{\circ} \mathrm{C}$. $\beta$-actin was used as the loading control. Subsequent to washing, the membranes were incubated with corresponding secondary antibodies (1:20,000 dilution; cat. no. 14709; Cell Signaling Technology, Inc.) for $60 \mathrm{~min}$ at $37^{\circ} \mathrm{C}$ and washed again. Signals were visualized using an enhanced chemiluminescence system (Bio-Rad Laboratories, Inc.), according to the manufacturer's protocols.

Proliferation and colony formation assays. Proliferation was analysed using the Cell Counting kit-8 reagent (CCK-8; Dojindo Molecular Technologies, Inc., Kumamoto, Japan), according to the manufacturer's protocols. Cells were cultured in 96-well plates at a density of 1,000 cells per well for 1, 2, 3, 4 and 5 days at $37^{\circ} \mathrm{C}$. The absorbance was subsequently measured at a wavelength of $450 \mathrm{~nm}$ on a spectrometer (Thermo Fisher Scientific, Inc.). To examine cell colonies, cells were cultured at a density of 200 cells/plate and grown for 10 days at $37^{\circ} \mathrm{C}$. The cells were then fixed with a methanol/acetic acid solution (3:1) for $15 \mathrm{~min}$ and stained with $0.5 \%$ crystal violet in methanol for $15 \mathrm{~min}$ at room temperature. Subsequent to staining, the visible colonies were counted under a light microscope.

Invitrowound-healing andinvasionassays.Forthewound-healing assay, $2 \times 10^{5}$ cells were cultured until confluent $(100 \%)$. The cell monolayer was then scratched with a $200-\mu 1$ pipette tip and the 
cells were washed three times with PBS. Fresh growth medium was subsequently added. The wounded area was monitored every $24 \mathrm{~h}$ under a microscope (DFC450; Leica Microsystems, Inc., Buffalo Grove, IL, USA) and the degree of wound healing induced by cell migration was quantified using Image-Pro Plus software 6.0 (Media Cybernetics, Inc., Rockville, MD, USA).

For cell invasion analysis, $5 \times 10^{4}$ cells in serum-free DMEM (GE Healthcare Life Sciences, Logan, UT, USA) were plated in the upper membrane of Transwell inserts $(8 \mathrm{~mm})$ coated with Matrigel (BD Biosciences, Franklin Lakes, NJ, USA). After $24 \mathrm{~h}$, the cells on the upper membrane were removed with a cotton swab and the cells that had invaded into the lower chamber with DMEM plus $10 \%$ FBS were stained with Wright-Giemsa. Images of cells were captured under a light microscope (DS-Ril Nikon; Nikon Corporation, Tokyo, Japan) and stained cells were counted by Image-Pro Plus software 6.0 (Media Cybernetics, Inc.).

Flow cytometry. Cells were seeded into 6-well plates, at $5 \times 10^{5}$ cells/well, and incubated for $24 \mathrm{~h}$. Cells were collected by trypsinization and washed once with PBS. The cells were fixed with $70 \%$ ethanol overnight at $20^{\circ} \mathrm{C}$, washed thoroughly with ice-cold PBS and incubated with a propidium iodide (PI) solution $(50 \mathrm{mg} / \mathrm{ml}$ RNase A, 0.1\% Triton X-100, $0.1 \mathrm{mmol} / \mathrm{l}$ EDTA and $50 \mathrm{mg} / \mathrm{ml} \mathrm{PI}$ ) for $\geq 30 \mathrm{~min}$ at $4^{\circ} \mathrm{C}$. The samples were evaluated with a FACSort instrument (BD Biosciences) and the data were analysed with ModFit LT 4.0 software (Verity Software House, Inc., Topsham, ME, USA).

Statistical analysis. All experiments were performed in triplicate and the represented data are derived from individual and separate experiments. Vector control cells (RNAi-Vector) were used as the control group in all experiments. All data were evaluated with SPSS version 20.0 (IBM Corp., Armonk, $\mathrm{NY}, \mathrm{USA}$ ) and the results are presented as the mean \pm standard deviation. Comparisons among groups were performed using an unpaired two-tailed Student's t-test. One-way analysis of variance was used for multiple comparisons to test for significant differences, followed by Tukey' spost hoc test. $\mathrm{P}<0.05$ was considered to indicate a statistically significant difference.

\section{Results}

SERINC2 mRNA is expressed at high levels in lung adenocarcinoma tissues. In our previous study (9), the expression levels of SERINC2 mRNA were evaluated by sequencing 59 pairs of lung adenocarcinoma tissues and adjacent healthy tissues. SERINC2 was expressed at significantly higher levels in lung adenocarcinoma tissues compared with those in corresponding adjacent healthy lung tissues (fold-change, 5.94; $\mathrm{P}<0.0001$; Fig. 1A). Previous analyses of published microarray datasets reported in Selamat et al (20) $(\mathrm{n}=116$; fold-change, 4.809; Fig. 1B), Okayama et al (21) ( $\mathrm{n}=246$; fold-change, 4.409; Fig. 1C) and Hou et al (22) ( $\mathrm{n=110}$; fold-change, 2.4971; Fig. 1D) indicated significantly higher expression levels of SERINC2 mRNA in lung adenocarcinoma tissues compared with those in healthy lung tissues.

SERINC2 silencing inhibits $H 1650$ and A549 proliferation. The lung adenocarcinoma H1650 and A549 cell lines were
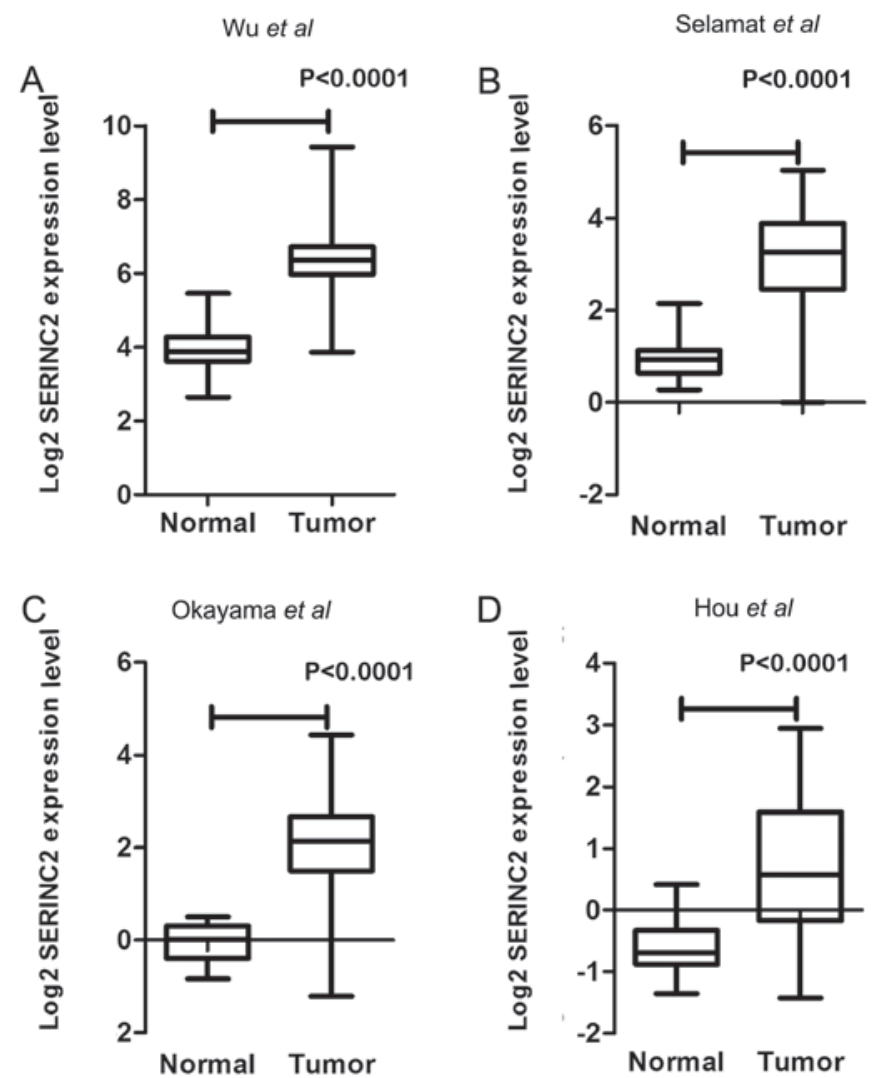

Figure 1. SERINC2 mRNA expression is significantly upregulated in primary human lung adenocarcinoma tissues. (A) SERINC2 mRNA expression levels in 59 pairs of lung adenocarcinoma tissues and adjacent normal lung tissues were measured in a study by Wu et al (9) using whole-genome sequencing. (B) SERINC2 mRNA expression levels in 58 pairs of lung adenocarcinoma tissues and adjacent normal lung tissues were assessed by Illumina HumanWG-6 v3.0 Expression BeadChip in a study by Selamat et al (20). (C) SERINC2 mRNA expression levels in lung adenocarcinoma tissue samples $(n=226)$ and adjacent normal lung tissue samples $(n=20)$ were evaluated by Affymetrix U133Plus2.0 arrays in a study by Okayama et al (21). (D) SERINC2 mRNA expression levels in lung adenocarcinoma tissue samples $(n=45)$ and adjacent normal lung tissue samples $(n=65)$ were assessed using Affymetrix U133Plus2.0 arrays in a study by Hou et al (22). SERINC2, serine incorporator 2.

selected as models to investigate the biological function of SERINC2. SERINC2 expression in H1650 and A549 cells was significantly suppressed by two shRNAs $(\mathrm{P}<0.05$; Fig. 2A and B). The effect of SERINC2 silencing on the proliferative activity of H1650 and A549 cells was assessed using a CCK- 8 assay, the results of which revealed that compared with the control groups, SERINC2-knockdown suppressed the viability of lung adenocarcinoma cell lines on days 3, 4 and 5 to a significantly greater extent $(\mathrm{P}<0.05$; Fig. $2 \mathrm{C})$. Furthermore, when SERINC2 expression was reduced in the H1650 and A549 cell lines, colony numbers were significantly decreased $(\mathrm{P}<0.05$; Fig. 2D). The effect of SERINC2-knockdown on the cell cycle was also determined. However, based on cell cycle analysis, there were no significant differences between the experimental and control groups (data not shown).

SERINC2 silencing inhibits the migration and invasion of H1650 and A549 cells. Wound healing and Transwell assays were performed to investigate the effect of SERINC2-knockdown on the migration and invasion activities 
A
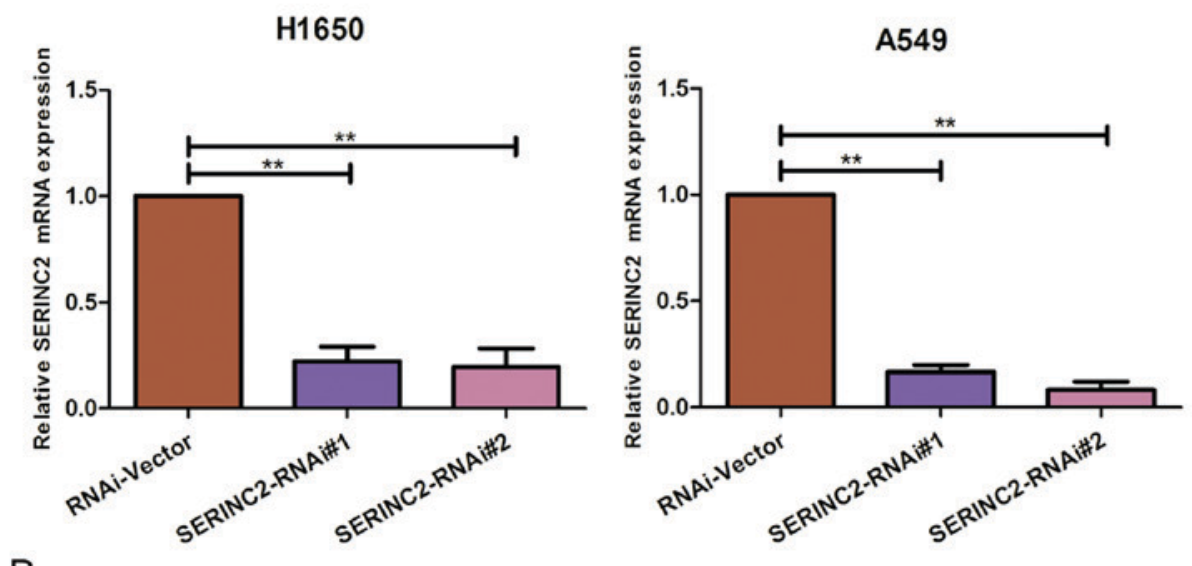

B

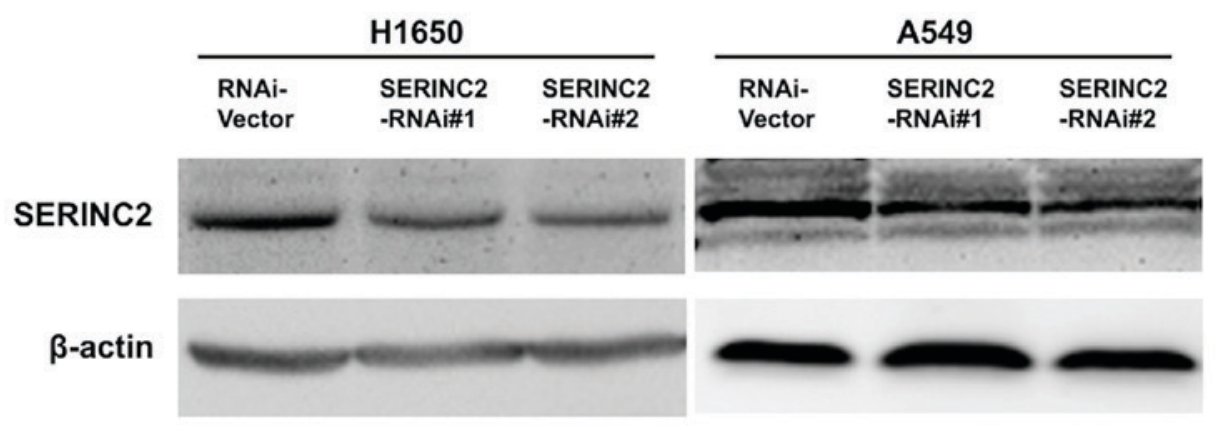

C
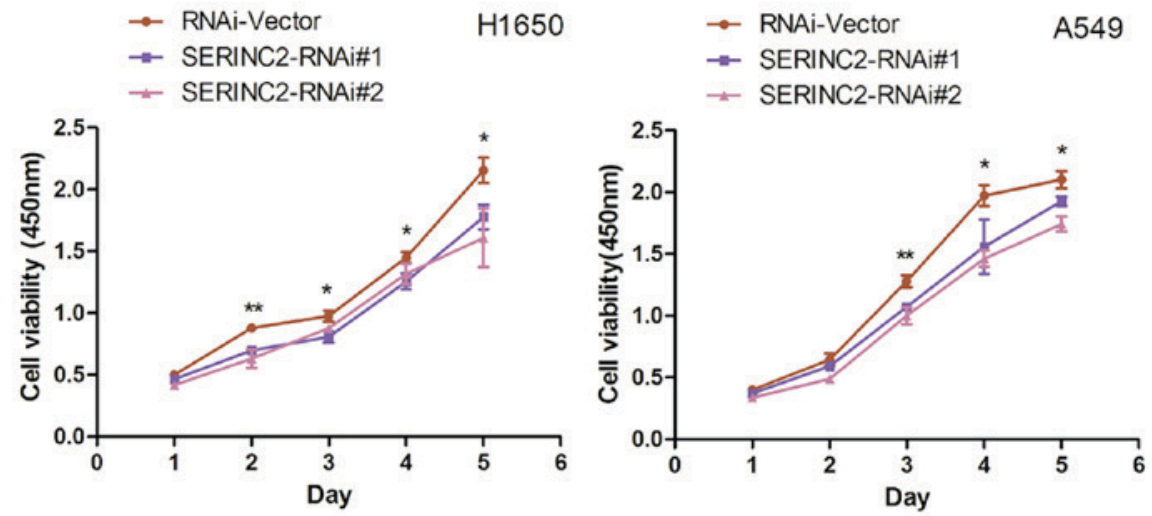

D
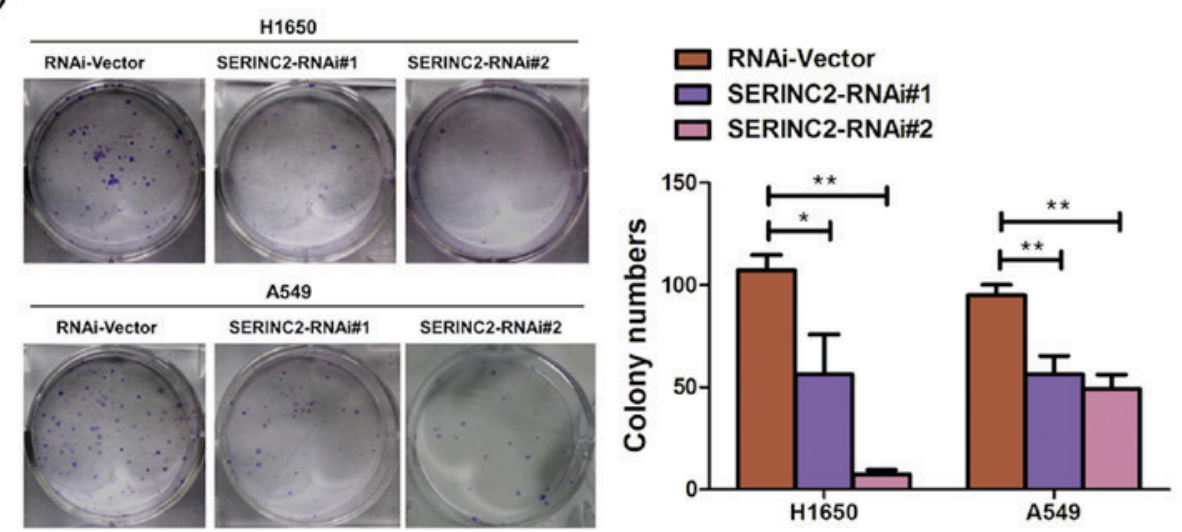

Figure 2. SERINC2-knockdown inhibits the proliferation of H1650 and A549 cells. Relative expression levels of SERINC2 mRNA in A549 (A) and H1650 (B) cells were significantly suppressed by two lentiviral-based short hairpin RNAs. (C) SERINC2-knockdown suppressed the viability of lung adenocarcinoma cell lines on days 3, 4 and 5 to a significantly greater extent compared with the control. (D) Colony numbers significantly decreased when SERINC2 expression was reduced in $\mathrm{H} 1650$ and A549 cell lines. ${ }^{*} \mathrm{P}<0.05$ and ${ }^{* *} \mathrm{P}<0.01$ compared with the control. RNAi, RNA interference; SERINC2, serine incorporator 2. 



Figure 3. SERINC2-knockdown significantly inhibits the migration and invasion of H1650 and A549 cells. (A) SERINC2-knockdown significantly inhibited the migration of $\mathrm{H} 1650$ and A549 cells. (B) SERINC2-knockdown significantly inhibited the invasion of H1650 and A549 cells. Error bars, $100 \mu \mathrm{m}$. ${ }^{*} \mathrm{P}<0.05$ and ${ }^{* *} \mathrm{P}<0.01$ compared with the control. RNAi, RNA interference; SERINC2, serine incorporator 2.

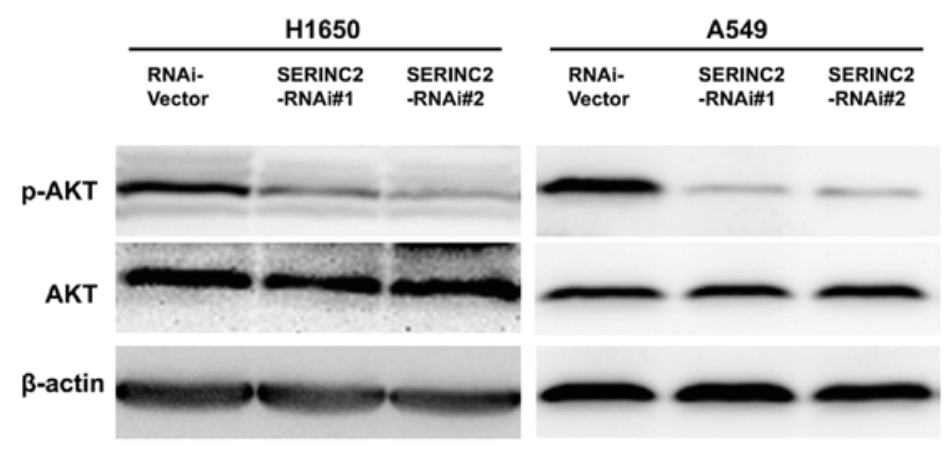

Figure 4. p-AKT is regulated by SERINC2. SERINC2-knockdown significantly reduced p-AKT expression levels in H1650 and A549 cells. $\beta$-Actin was used as the internal control. p, phosphorylated; SERINC2, serine incorporator 2; RNAi, RNA interference; AKT, serine/threonine kinase.

of H1650 and A549 cells, respectively. SERINC2-knockdown significantly inhibited wound healing $(\mathrm{P}<0.05$; Fig. $3 \mathrm{~A})$, as well as invasive capacity $(\mathrm{P}<0.01 ;$ Fig. 3B).

Expression levels of phosphorylated-protein kinase $B$ $(p-A K T)$ are altered by SERINC2-knockdown. p-AKT protein expression levels were quantified to investigate the possible underlying mechanisms of SERINC2 activity. Levels of p-AKT were significantly decreased when SERINC2 was knocked down in the H1650 and A549 cells. However, a minimal effect was observed on total AKT (Fig. 4). 


\section{Discussion}

In the present study, SERINC2-knockdown using lentiviralbased shRNAs inhibited the proliferation, migration and invasion of lung adenocarcinoma cells. In addition, expression levels of p-AKT were significantly decreased upon SERINC2-knockdown.

In our previous study, the whole-genome sequencing approach was employed to distinguish DEGs between lung adenocarcinoma tissues and adjacent healthy tissues (9). SERINC2 was identified as a DEG that was significantly upregulated in lung adenocarcinoma. A previous analysis of published microarray datasets also indicated SERINC2 mRNA to be expressed at significantly higher levels in lung adenocarcinoma tissues compared with those in healthy lung tissues (20-22). Player et al (18) observed high SERINC2 expression levels in the bladder, kidney and muscle; moderate expression in the stomach, liver, skin, placenta and ovary, and minimal expression in the brain, spleen and heart. Based on RT-PCR and in situ hybridization data, an increase in SERINC2 expression was reported in NSCLC compared with that in healthy lung tissues (18). Regardless, the role of SERINC2 in cancer requires further investigation.

H1650 cells are derived from metastatic lung adenocarcinoma $(23,24)$ and A549 cells are a lung adenocarcinoma cell line $(25,26)$. These lung adenocarcinoma cell lines were utilized to investigate the role of SERINC2 in the present study, and it was indicated that SERINC2 silencing inhibited proliferation based on CCK-8 and colony formation assays . SERINC1-knockdown has been previously reported to result in cell cycle arrest and the inhibition of proliferation in hepatocellular carcinoma cell lines (17). According to the data of the present study, SERINC2 silencing inhibits the migration and invasive abilities of $\mathrm{H} 1650$ and A549 cells. SERINC1 and SERINC2 belong to the SERINC family of transmembrane proteins that incorporate serine into phosphatidylserine and sphingolipids (10). Endoplasmic reticulum membranes are enriched with SERINC proteins, directly binding to serine palmitoyltransferase, the key enzyme in sphingolipid biosynthesis. Therefore, SERINC proteins serve an essential role in regulating the biosynthesis of lipids, including phosphatidylserine and sphingolipids (10). Bavituximab is a phosphatidylserine-targeting monoclonal antibody representing a novel strategy in cancer therapy (11). As sphingolipid synthesis, including ceramide and sphingosine-1-phosphate-mediated pathways, has been reported to serve a vital role in cancer development and progression (16), SERINC2 may also be important in cancer development and progression. Overall, the molecular underlying mechanism of lipid biosynthesis requires further investigation.

Sterol regulatory element-binding proteins (SREBPs) are transcription factors associated with cholesterol and fatty acid synthesis (27), and SREBP-1a causes G1 cell cycle arrest following the accumulation of cyclin-dependent kinase inhibitors, including p27, p21 and p16 (28). As indicated in the study by Ren et al (17), downregulation of SERINC1 expression enhanced expression of SREBPs and p21, resulting in impaired cell cycle progression of hepatocellular carcinoma. However, SERINC2-knockdown was not reported to cause significant differences in the cell cycle in lung adenocarcinoma cell lines.
In addition, significant changes in expression of the low-density lipoprotein receptor gene, a target of SREBPs $(27,29)$, were not observed when SERINC2 expression was knocked down in lung adenocarcinoma cells (data not shown). Therefore, further research on SREBPs was not conducted. Differences between SERINC2 and SERINC1 have been observed, presumably due to their different underlying mechanisms in regulating cancer.

Phosphatidylinositol 3-kinase (PI3K)/AKT signalling serves an important role in the proliferation, survival, differentiation and motility of cancer cells. It has been reported that constitutive activation of this pathway is a common feature of human types of cancer and is frequently the outcome upon molecular alterations of key components of the signalling cascade (30). The focus of such studies has contributed to the development of novel molecule-targeted therapies, including PI3K inhibitors (31). In the present study, SERINC2-knockdown significantly decreased AKT phosphorylation. According to the study by Huang et al (32), phosphatidylserine is a critical modulator of AKT activation. Furthermore, it was indicated in the study by Zhang et al (33) that phosphatidylserine on the neuronal membrane may facilitate membrane translocation of AKT in a phosphoinositide-3,4,5-trisphosphate-dependent manner, thereby enhancing the subsequent phosphorylation and activation of AKT. As the SERINC family of transmembrane proteins has been reported to facilitate the incorporation of serine into phosphatidylserine (10), SERINC2 may affect the pathway by modulating lipid synthesis. Therefore, SERINC2-knockdown has been suggested for inhibiting the proliferative, migratory and invasive abilities of cells, and it may be associated with the regulation of the PI3K/AKT pathway. However, further investigations are required to confirm whether SERINC2-knockdown affects phosphatidylserine synthesis and directly regulates the PI3K/AKT pathway. In addition, in vivo studies of the role of SERINC2 are required. Despite these limitations, the present study provides a unique perspective for evaluating the role of SERINC2 in lung adenocarcinoma.

In conclusion, the present study provides evidence that the expression of SERINC2 mRNA is significantly elevated in tumour tissues derived from patients with lung adenocarcinoma. Knockdown of SERINC2 expression using a lentiviral-based shRNA inhibited the proliferation, migration and invasion of lung adenocarcinoma cells, which may be associated with the PI3K/AKT pathway. Based on the findings, SERINC2 is required for lung adenocarcinoma progression. The molecular underlying mechanism warrants further investigation.

\section{Acknowledgements}

Not applicable.

\section{Funding}

No funding was received.

\section{Availability of data and materials}

The datasets used and/or analyzed during the present study are available from the corresponding author on reasonable request. 


\section{Authors' contributions}

JianH, YZ and DX were responsible for the conceptualization. YZ, DX and HH performed the methodology. YZ, HP and YC performed the software analysis. JianH and DX were responsible for the validation. YZ, DX, JiaxH and WY performed the formal analysis and interpretation of data. YZ, DX and HH conducted the investigation. JianH was responsible for the resources and YZ for the data curation. The manuscript writing (preparation of the original draft) was performed by YZ and DX. Manuscript preparation (writing, review and editing) was completed by $\mathrm{YZ}$, DX, JianH, JiaxH, HP, WY and YC. The present study was supervised by JianH and administrated by YZ and DX. Final approval of the version was provided by all authors.

\section{Ethics approval and consent to participate}

Not applicable.

\section{Patient consent for publication}

Not applicable.

\section{Competing interests}

The authors declare that they have no competing interests.

\section{References}

1. Torre LA, Bray F, Siegel RL, Ferlay J, Lortet-Tieulent J and Jemal A: Global cancer statistics, 2012. CA Cancer J Clin 65: 87-108, 2015.

2. Molina JR, Yang P, Cassivi SD, Schild SE and Adjei AA: Non-small cell lung cancer: Epidemiology, risk factors, treatment, and survivorship. Mayo Clin Proc 83: 584-594, 2008.

3. Goldstraw P, Ball D, Jett JR, Le Chevalier T, Lim E, Nicholson AG and Shepherd FA: Non-small-cell lung cancer. Lancet 378: 1727-1740, 2011.

4. Imielinski M, Berger AH, Hammerman PS, Hernandez B, Pugh TJ, Hodis E, Cho J, Suh J, Capelletti M, Sivachenko A, et al: Mapping the hallmarks of lung adenocarcinoma with massively parallel sequencing. Cell 150: 1107-1120, 2012.

5. Paez JG, Jänne PA, Lee JC, Tracy S, Greulich H, Gabriel S, Herman P, Kaye FJ, Lindeman N, Boggon TJ, et al: EGFR mutations in lung cancer: Correlation with clinical response to gefitinib therapy. Science 304: 1497-1500, 2004

6. Kwak EL, Bang YJ, Camidge DR, Shaw AT, Solomon B, Maki RG, Ou SH, Dezube BJ, Jänne PA, Costa DB, et al: Anaplastic lymphoma kinase inhibition in non-small-cell lung cancer. N Engl J Med 363: 1693-1703, 2010.

7. Roberts PJ and Stinchcombe TE: KRAS mutation: Should we test for it, and does it matter. J Clin Oncol 31: 1112-1121, 2013.

8. Bergethon K, Shaw AT, Ou SH, Katayama R, Lovly CM, McDonald NT, Massion PP, Siwak-Tapp C, Gonzalez A, Fang R, et al: ROS1 rearrangements define a unique molecular class of lung cancers. J Clin Oncol 30: 863-870, 2012.

9. Wu K, Zhang X, Li F, Xiao D, Hou Y, Zhu S, Liu D, Ye X, Ye M, Yang J, et al: Frequent alterations in cytoskeleton remodelling genes in primary and metastatic lung adenocarcinomas. Nat Commun 6: 10131, 2015.

10. Inuzuka M, Hayakawa $M$ and Ingi T: Serinc, an activity-regulated protein family, incorporates serine into membrane lipid synthesis. J Biol Chem 280: 35776-35783, 2005.

11. Digumarti R, Bapsy PP, Suresh AV, Bhattacharyya GS, Dasappa L, Shan JS and Gerber DE: Bavituximab plus paclitaxe and carboplatin for the treatment of advanced non-small-cell lung cancer. Lung Cancer 86: 231-236, 2014.

12. Yin Y, Huang $X$, Lynn KD and Thorpe PE: Phosphatidylserine-targeting antibody induces M1 macrophage polarization and promotes myeloid-derived suppressor cell differentiation. Cancer Immunol Res 1: 256-268, 2013.
13. Bondanza A, Zimmermann VS, Rouvere-Querini P, Turnay J, Dumitriu IE, Stach CM, Voll RE, Gaipl US, Bertling W, Pöschl E, et al: Inhibition of phosphatidylserine recognition heightens the immunogenicity of irradiated lymphoma cells in vivo. J Exp Med 200: 1157-1165, 2004.

14. Kok JW and Sietsma H: Sphingolipid metabolism enzymes as targets for anticancer therapy. Curr Drug Targets 5: 375-382, 2004.

15. Proia RL and Hla T: Emerging biology of sphingosine-1-phosphate: Its role in pathogenesis and therapy. J Clin Invest 125: 1379-1387, 2015

16. Ogretmen B and Hannun YA: Biologically active sphingolipids in cancer pathogenesis and treatment. Nat Rev Cancer 4: 604-616, 2004.

17. Ren WH, Yang CY, Yang XM and Yu L: siRNA-mediated knockdown of hTDE2 retards cell cycle progression through transcriptional activation of p21. Oncol Rep 31: 1314-1322, 2014.

18. Player A, Gillespie J, Fujii T, Fukuoka J, Dracheva T, Meerzaman D, Hong KM, Curran J, Attoh G, Travis W and Jen J: Identification of TDE2 gene and its expression in non-small cell lung cancer. Int J Cancer 107: 238-243, 2003.

19. Livak KJ and Schmittgen TD: Analysis of relative gene expression data using real-time quantitative PCR and the 2(-Delta Delta C(T)) method. Methods 25: 402-408, 2001.

20. Selamat SA, Chung BS, Girard L, Zhang W, Zhang Y, Campan M, Siegmund KD, Koss MN, Hagen JA, Lam WL, et al: Genome-scale analysis of DNA methylation in lung adenocarcinoma and integration with mRNA expression. Genome Res 22: 1197-1211, 2012.

21. Okayama H, Kohno T, Ishii Y, Shimada Y, Shiraishi K, Iwakawa R, Furuta K, Tsuta K, Shibata T, Yamamoto S, et al: Identification of genes upregulated in ALK-positive and EGFR/KRAS/ALK-negative lung adenocarcinomas. Cancer Res 72: 100-111, 2012.

22. Hou J, Aerts J, den Hamer B, van Ijcken W, den Bakker M, Riegman P, van der Leest C, van der Spek P, Foekens JA, Hoogsteden HC, et al: Gene expression-based classification of non-small cell lung carcinomas and survival prediction. PLoS One 5: e10312. 2010.

23. NCI-H1650 [H-1650,H1650] (ATCC ${ }^{\circledR}$ CRL-5883 $\left.{ }^{\mathrm{TM}}\right)$. https://www. atcc.org/ /ps/CRL-5883.ashx.

24. NCI-Navy Medical Oncology Branch cell line supplement. J Cell Biochem Suppl 24: 1-291, 1996.

25. Sexton DW,Blaylock MG and Walsh GM: Human alveolar epithelial cells engulf apoptotic eosinophils by means of integrin- and phosphatidylserine receptor-dependent mechanisms: A process upregulated by dexamethasone. J Allergy Clin Immunol 108: 962-969, 2001.

26. Boussat S, Eddahibi S, Coste A, Fataccioli V, Gouge M, Housset B, Adnot S and Maitre B: Expression and regulation of vascular endothelial growth factor in human pulmonary epithelial cells. Am J Physiol Lung Cell Mol Physiol 279: L371-L378, 2000.

27. Shimano H: Sterol regulatory element-binding proteins (SREBPs): Transcriptional regulators of lipid synthetic genes. Prog Lipid Res 40: 439-452, 2001.

28. Nakakuki M, Shimano H, Inoue N, Tamura M, Matsuzaka T, Nakagawa Y, Yahagi N, Toyoshima H, Sato R and Yamada N: A transcription factor of lipid synthesis, sterol regulatory element-binding protein (SREBP)-1a causes G(1) cell-cycle arrest after accumulation of cyclin-dependent kinase (cdk) inhibitors. FEBS J 274: 4440-4452, 2007.

29. Streicher R, Kotzka J, Muller-Wieland D, Siemeister G, Munck M, Avci H and Krone W: SREBP-1 mediates activation of the low density lipoprotein receptor promoter by insulin and insulin-like growth factor-I. J Biol Chem 271: 7128-7133, 1996.

30. Downward J: Targeting RAS signalling pathways in cancer therapy. Nat Rev Cancer 3: 11-22, 2003.

31. Bedard PL, Tabernero J, Janku F, Wainberg ZA, Paz-Ares L, Vansteenkiste J, Van Cutsem E, Pérez-García J, Stathis A, Britten CD, et al: A phase Ib dose-escalation study of the oral pan-PI3K inhibitor buparlisib (BKM120) in combination with the oral MEK1/2 inhibitor trametinib (GSK1120212) in patients with selected advanced solid tumors. Clin Cancer Res 21: 730-738, 2015.

32. Huang BX, Akbar M, Kevala K and Kim HY: Phosphatidylserine is a critical modulator for Akt activation. J Cell Biol 192: 979-992, 2011.

33. Zhang W, Liu J, Hu X, Li P, Leak RK, Gao Y and Chen J: n-3 polyunsaturated fatty acids reduce neonatal hypoxic/ischemic brain injury by promoting phosphatidylserine formation and Akt signaling. Stroke 46: 2943-2950, 2015.

This work is licensed under a Creative Commons Attribution-NonCommercial-NoDerivatives 4.0 International (CC BY-NC-ND 4.0) License. 